\title{
Corrupción administrativa y terrorismo Un distanciamiento considerable entre intereses y publicaciones
}

\author{
Administrative Corruption and Terrorism: \\ A Considerable Distancing between Interests and Publications
}

Solon Bevilacqua y John Edward Neira Villena*

Resumen: Este estudio investiga la corrupción administrativa (CA) que sufrió alteraciones significativas en los últimos veinte años, pero los intereses existentes aún están lejos de ser atendidos. A partir del análisis de clúster para 253 artículos publicados (double review) se evidenciaron oportunidades y brechas en el trabajo de la ciencia que investiga la CA en el mundo. A partir de un análisis de contenido y de correspondencia, se comprobó que la violencia extrema, como el terrorismo y el narcotráfico, no se analizan en la agenda de la investigación de la CA; "crimen y punición" forman un enlace histórico en la teoría de la CA, pero raramente son investigados en el nivel del comportamiento humano y de Data Science; hay una incidencia significativa de publicaciones relacionadas con las prácticas de combate a la CA, mientras que los estudios que investigaron externalidades positivas y negativas para ese tema son escasos.

Palabras clave: problemas sociales, administración pública, delitos, fraude, corrupción.

Abstract: Research investigating administrative corruption (AC) has changed significantly in the last twenty years, but the existing interests are still far from being met. From the cluster analysis for 253 published articles (double review) were shown opportunities and gaps in the work of science that investigates the AC in the world. From a content analysis and correspondence analysis, it was found that extreme violence, such as terrorism and drug trafficking, are not discussed in AC research agenda; "crime and punishment" form a historical link in AC theory, but they are often investigated at the level of human behaviour and Data Science;

*Solon Bevilacqua es profesor efectivo en la Faculdade de Ciências e Tecnologia da Universidade Federal de Goiás-FCT/UfG, Rua Mucuri S/N, Setor Conde dos Arcos-Campus Aparecida de Goiânia CEP: 74.968755, Aparecida de Goiânia, Goiás, Brasil. Tel: +55 (62) 3209 6550. Correo-e: solon@ufg.br. orcid: https:// orcid.org/0000-0002-0050-3527. John Edward Neira Villena también es profesor efectivo en la Faculdade de Ciências e Tecnologia da Universidade Federal de Goiás-FCT/UfG. Tel: +55 (62) 3209 6550. Correo-e: johnneirav@ufg.br. oRCID: https://orcid.org/0000-0003-2281-6505.

Artículo recibido el 8 de agosto de 2020 y aceptado para su publicación el 12 de marzo de 2021. 
there is a significant incidence of publications related to AC combat practices. However, for this subject few studies have explored both positive and negative externalities.

Keywords: social problems, public administration, crimes, fraud, corruption.

\section{INTRODUCCIÓN}

$\neg \mathrm{n}$ el periodo entre 1964 y 2020, se publicaron cerca de 250 artículos, en el _sistema double review, involucrando el tema cA. Cuestiones como fraude contable, indicadores de gobernanza, medio ambiente y políticas de combate a la corrupción estuvieron presentes en esas investigaciones científicas. La mayoría de las publicaciones atendieron detalles ampliados de los problemas enfrentados por la administración pública, tales como fraude contable, soborno, políticas anticorrupción, mercado financiero, entre otros. Algunos puntos importantes pasaron desapercibidos, como aspectos comportamentales que motivan la corrupción o aún la ligación estrecha entre el terrorismo y la corrupción en las administraciones. En la mayoría de los casos los intereses de los lectores no fueron atendidos, los investigadores no estudiaron la estructura ni el medio en que se forma la corrupción, como crisis económicas, religión y terrorismo.

¿Como están definidos los clústeres de investigación para el tema "corrupción administrativa” en el periodo comprendido entre 2001 y 2019? Una búsqueda para la palabra clave administrative cormption realizada a partir de la herramienta Google Trends evidenció que hay un descompás entre lo que se busca en la gran red y lo que está siendo publicado como ciencia. Se emplearon herramientas de análisis de datos para plotear gráficos de clúster para identificar tendencias, oportunidades y brechas entre las investigaciones realizadas en los últimos cincuenta años.

En este artículo se buscó medir la brecha entre oferta académica y demanda por el tema corrupción administrativa. La importancia de esta búsqueda reside en proponer un nuevo orden de estudios relacionados con la corrupción. De esta manera, estudios que no están alineados con el interés del lector se colocan en segundo plano, para evitar desperdicio de esfuerzos. La investigación evidenció que la atención de los investigadores está orientada a la administración pública y a las políticas anticorrupción, mientras que las búsquedas en sitios como el Google registran interés convergente en corrupción y terrorismo, algo muy distante de lo que se viene publicando. Además, se constató que el concepto de terrorismo relacionado con la corrupción administrativa está equivocado. El terrorismo financiado por la corrupción no es solamente teología, ideología, rabia o psicosis, sino que es un mal que sirve a intereses políticos y a una lucha por la causa (altruismo) (Merari, 1993). 
Los resultados son teóricos y aplicados naturalmente. A partir de una búsqueda teórica en bases de investigación fue posible orientar tendencias para clústeres de investigación y sugerir readecuaciones para las demandas de estudios que abarcan cA y terrorismo.

El resto de este artículo está estructurado de la siguiente forma: corrupción administrativa y terrorismo; aspectos metodológicos y análisis de datos. Los resultados se analizan de forma cualitativa y, al final del estudio, se presentan consideraciones y una agenda de investigación.

\section{CORRUPCIÓN ADMINISTRATIVA}

Una de las mayores dificultades enfrentadas en las investigaciones sobre corrupción administrativa es su propia definición. Hay un consenso acerca de que la corrupción se refiere a actos en los cuales el poder del cargo público se usa para la codicia personal de una manera que viola las reglas del juego. Algunos investigadores no consideran fraude, lavado de dinero, comercio de drogas y operaciones en el mercado negro como ca porque no involucran el uso del poder público. Mientras que tales actividades generalmente involucran funcionarios públicos y políticos para que esas operaciones puedan sobrevivir y, por lo tanto, esas actividades raramente prosperan sin corrupción generalizada. Otro punto refuerza la cuestión: inclusive en sus formas más simples, esos actos pueden influir las políticas del gobierno (Jain, 2001; Ugalde, 2018). También es posible crear niveles para una red de corrupción, divididos en redes caníbales, redes de exploradores, redes parásitas y redes monopolísticas. La idea se presenta interesante cuando provoca un entendimiento de la interconectividad entre los niveles de corrupción, que generalmente son vistas como puntos aislados y no como redes (Jancsics y Jávor, 2012).

Los orígenes de la corrupción administrativa parecen estar relacionados con los funcionarios públicos que no actúan de acuerdo con el interés público, pero usan sus posiciones para beneficiarse a ellos mismos y a sus familias, amigos o asociados. En esta situación no solo se pierden recursos financieros, sino también la esperanza y la confianza de la población, puntos difíciles de ser previstos (T. Vian, 2008). Se trata de una situación que implica entender los factores ocultos y prácticas veladas en toda organización, principalmente cuando la corrupción involucra soborno (Del Castillo, 2001).

Para que ese ambiente exista deben estar presentes tres elementos: $a$ ) alguien debe tener poder discrecional, una autoridad para proyectar reglas y también para administrarlos; $b$ ) debe haber rentas asociadas a ese poder; $c$ ) el sistema 
jurídico debe ofrecer una probabilidad de detección o penalidad suficientemente baja para las irregularidades (Jain, 2001). Los dos primeros elementos se combinan para crear incentivos a la corrupción y el tercero actúa como un impedimento. Además, todos deberán tener la misma probabilidad de ser punidos; debe haber independencia del poder judicial de los políticos; la ejecución de los corruptos debe ocurrir con efectividad y finalmente debe ocurrir una alta probabilidad de un corrupto ser descubierto por el sistema anticorrupción (Becker, 1968).

Hasta mediados de la década de 1950 el tema corrupción administrativa no era pauta prioritaria en las investigaciones de ciencias sociales, quizá porque privaba la visión de que los países desarrollados, principalmente Estados Unidos, eran países morales (Werner, 1983). Ese escenario rápidamente fue alterado, cuando la corrupción administrativa comenzó a ser percibida como algo endémico, que debería ser tratado con rigor, ya que sus consecuencias podrían generar una serie de problemas, como inviabilizar las transiciones políticas y económicas, minar la capacidad y la legitimidad del Estado, exacerbar la pobreza (Hurwitz y Huang, 2014), crear crisis de seguridad nacional (Factor y Kang, 2015; Shelley, 2004), llevar a la decadencia, colocar las políticas de un gobierno contra una nación, desperdiciar recursos nacionales, reducir la eficiencia de los gobiernos en la administración de los asuntos del país, aumentar la indiferencia y la ineficiencia en la sociedad, destruir las creencias y los valores morales de la sociedad, aumentar los costos de implementación de proyectos y dificultar el crecimiento de la competitividad (Mousavi y Pourkiani, 2013). Además de esas cuestiones, la corrupción administrativa también es capaz de minar el estado de derecho y llevar a otras amenazas de la seguridad nacional, como crimen extra nacional (Afzali, 2011).

La corrupción en el servicio público debe combatirse con una combinación de incentivos y sanciones que favorezcan normas profesionales de conducta. Una salida natural parece ser minimizar la posibilidad de que surjan conflictos entre los intereses privados y las obligaciones públicas para aquellos que ocupan cargos públicos. Sería un pasaje natural crear reglas claras y transparentes como elementos claves para la búsqueda de esta postura anticorrupción (Bertock, 2001).

También es importante definir cuáles controles anticorrupción deben emplearse para combatir las mayores pérdidas de eficiencia generada por el comportamiento corrupto. Procedimientos contables y auditorías son probablemente las medidas con mayor potencial para promover la eficiencia pública (Jacobs y Anechiarico, 2001). La cuestión demanda nuevos entendimientos para problemas 
antiguos. En consecuencia, no se trata apenas de practicar una cultura de sigilo para combatir la corrupción, sino de adoptar también medidas que privilegien la transparencia (Santin y Frizon, 2020).

Acompañar la corrupción y sus nuevas formas es fundamental para mantener actualizadas las estrategias de combate y mitigación de la cA. Una de las brechas evidenciadas en las publicaciones relacionadas con el tema es la nueva apariencia que la corrupción asumió en los últimos veinte años. La corrupción administrativa se aproximó del crimen organizado mundial. Tanto el narcotráfico como el terrorismo son en parte sustentados por la corrupción pública y un círculo vicioso CA-crimen organizado-CA se instaló y corrompió la estructura social, económica y política de muchos países en vías de desarrollo.

Esta cuestión no puede simplificarse en virtud de que el terrorismo y la corrupción aumentaron significativamente después de los atentados del 11 de septiembre. Después de esta tragedia, Estados Unidos y sus aliados emprendieron una jornada contra el terrorismo mundial. Esa acción, además de no surtir efecto, aumentó en 45 por ciento el número de muertes relacionadas con el terrorismo mundial, financiado por la corrupción. Al-Qaeda no consiguió otro día tan devastador como los atentados de septiembre negro; sin embargo, los atentados en Madrid, Londres, Bali, Sinaí, y Omán; los ataques en Turquía, Chechenia y Mumbai, y la guerra aún en curso en Irak, Afganistán y Paquistán, ofrecen evidencias nítidas de que el terrorismo global está empeorando (Merari, 1991).

Lo anterior se comprobó en el análisis de clúster de esta investigación, evidenciando que ese es uno de los grandes intereses en las búsquedas en sitios de investigación, mientras que la comunidad científica se encuentra alerta para problemas periféricos.

\section{Corrupción administrativa y su relación con el terrorismo}

La corrupción se presenta como la principal amenaza al desarrollo económico, para la búsqueda de estabilidad política y la paz. También es vista en el contexto mundial como el agente promotor de la violencia, con el estatus de crimen organizado y terrorismo. Principalmente en los países en vías de desarrollo — debido a la diseminación de la corrupción y a la destinación incorrecta de recursos - ese flagelo gana fuerza, ya sea a partir de la producción y distribución del terror, proveniente de ideologías políticas y religiosas, o a partir de la producción y distribución de drogas ilícitas. Las principales fuentes de financiamiento del terrorismo vienen de la corrupción, robo, secuestro, tráfico de drogas, tráfico de armas e inmigrantes ilegales (Boussiga y Ghdamsi, 2016). El terrorismo frecuen- 
temente se coloca contra el Estado. Muchas veces contra el que lo financia con la corrupción (Crenshaw, 1981).

De una forma simplificada, existen dos grupos que nacieron de la corrupción y después se volvieron células de terror. Un tipo está interesado en obtener y retener recursos y poder para sí mismo y sus líderes. Para este grupo, ayudar a la población es una forma de obtener poder y riqueza. El segundo tipo está interesado en mejorar la suerte del pueblo por medio de la derrota política o militar del gobierno. Para este grupo, el bienestar de la población es una meta importante, independientemente del poder y la riqueza. Ambos grupos son semejantes en que actúan en la falla del Estado en solucionar los problemas de su pueblo (Magouirk, 2008).

El terrorismo y el crimen organizado son las dos mayores variantes de violencia para los países en vías de desarrollo, pero es posible enumerar otras tres mutaciones, más insidiosas, capaces de infligir daños a una nación: la burocracia, una ineficiencia que detiene los procesos administrativos; el clientelismo, que se traduce en favoritismo en los nombramientos políticos y alquiler de contratos; el soborno, que a veces en gran escala es endémico en muchas partes del mundo (Poole-Robb y Bailey, 2002). Cabe resaltar que lo rotulado como corrupción en los países desarrollados no es el mismo fenómeno de corrupción en los países en vías de desarrollo (Mungiu-Pippidi, 2013; Mungiu-Pippidi y Dusu, 2011).

En este artículo el terrorismo asume una forma de lucha en vez de aberración social o política (Merari, 1993). El terrorismo, la corrupción, la política y las religiones extremistas andan juntas, actuando alineados para conseguir un objetivo violento. Organizaciones como el Hezbollah actualmente es colega del gobierno de coalición del Líbano, con poder de veto; el Hamas construye la milicia más poderosa de Palestina, venció las elecciones nacionales en enero de 2006 y derrotó decenas de millares de fuerzas de seguridad palestinas para asumir el control militar de la Franja de Gaza en junio de 2007. En esas prácticas el objetivo se consiguió con unidad. La mayor parte de los recursos que financian tales operaciones provienen de instituciones de caridad religiosas. La eficacia de AlQaeda en Irak es menos sorprendente que la del Talibán, Hezbollah o Hamas, pero consiguió una evolución rápida de una organización religiosa benigna a una milicia potente con poder político sustancial (Berman, 2011).

Se trata de un camino natural para el terror, comenzar como una organización religiosa benigna, pasar por una organización de milicia militar y enseguida infiltrarse con actividad política influyente. Esas cuestiones se clarificaron cuando el investigador Ariel Merari entrevistó cuidadosamente familias y amigos de atacantes suicidas palestinos e incluso entrevistando a los propios atacantes suicidas 
que no consiguieron detonar sus cintos explosivos y fueron capturados en ese tipo de misión. El investigador concluye que factores como teología, ideología, rabia o psicosis son importantes para la ejecución de actos terroristas. Lo más importante es el altruismo por la causa. Talvez ese punto esclarezca el aumento de los actos terroristas postseptiembre negro (Merari, 1991, 1993; Nehemia y Merari, 1985).

En 2012 un estudio centrado en corrupción, terrorismo, libertad económica y crecimiento investigó si el terrorismo tiene mayor probabilidad de nacer en regímenes marcados por la corrupción. La muestra tenía países como Argelia, Bahréin, Egipto, Irán, Jordania, Líbano, Libia, Marruecos, Qatar, Arabia Saudita, Siria, Tunicia y Yemen. Se encontró que cuanto más corruptos son los gobiernos, mayor es la probabilidad de que los grupos extremistas de esos países se involucren en actividades terroristas. Por lo tanto, la motivación propuesta para el terrorismo como "venganza contra la corrupción" se ajustó adecuadamente a los datos, ya que refleja el círculo vicioso que involucra la corrupción administrativa y el terrorismo (Factor y Kang, 2015). En este estudio en específico el terrorismo y la corrupción fueron investigados en el Norte de África y Medio Oriente, con estrechas conexiones ideológicas y religiosas. En otras regiones, como América Latina, los orígenes para esta relación son otras. En América Latina impera una variante de la relación terrorismo - corrupción, materializada en el narcoterrorismo.

En esta región los ingredientes necesarios para producir drogas ilícitas pueden encontrarse en países como Ecuador, Perú y Bolivia, los productos químicos vienen de Brasil; las organizaciones poderosas y sus laboratorios están en Colombia. En el contexto de narcóticos, no hay una gran diferencia entre países: prácticamente todos los países de América Latina están involucrados de alguna manera, en alguna escala. Cada país participa con su contribución específica: de la producción a la transformación para el procesamiento y, finalmente, la distribución. En estas regiones se establecieron lazos notablemente fuertes a lo largo de los años entre los narcotécnicos y las autoridades. Los gobiernos están involucrados en todos los niveles: del condado, al estado y al federal, abarcando hasta el sistema judiciario. Aquí se registra una brecha importante en los estudios sobre corrupción: se usa el mismo abordaje y el mismo punto de vista para las dos modalidades de crimen organizado. No hay, por ejemplo, una metodología que investigue las dos variantes con objetivo en el lavado de dinero y el nacimiento de empresas ficticias (Ajayi y Millard, 1997).

La oportunidad de investigación se relaciona con un mercado significativo, donde impera la ca pulverizada en la estructura de la sociedad y en macroescala. 
Una parte significativa de los lucros obtenidos con el comercio de drogas ilícitas remunera una gran red de corrupción mundial. En Europa y Estados Unidos, cada 100 dólares de cocaína recompensa con 0.40 centavos al productor y otros 99.60 dólares para el crimen y la corrupción en los países productores y distribuidores (Clutterbuck, 1995). Los resultados de este estudio evidencian que los países del Tercer Mundo, Medio Oriente y América Latina son productores de corrupción porque también son piezas del sistema que sustenta el círculo de la corrupción y la violencia.

Hasta inicios de los años 2000, eran raros los artículos científicos que investigaban el terrorismo y casos aislados investigaron el círculo vicioso de la corrupción y de la violencia. A partir de ese periodo, esta estrecha relación asume contornos complejos en la promoción del terrorismo mundial (Angel Olofinbiyi, 2020; Esoimeme, 2020; Gächter y Schulz, 2016).

Cuatro de esos raros ejemplos estudiaron la postocupación norteamericana de 2003 en Basora, Irak (Al-Jundi, 2019); los impactos económicos mundiales con el terrorismo (Al-Jundi et al., 2019); los recursos del comercio ilícito de diamantes que alimentan conflictos armados en los países africanos. En este último caso, en específico, los resultados del estudio crearon un nexo entre el tráfico ilícito de diamantes y las operaciones de Al-Qaeda en mercados negros en Kenia, Tanzania, Burkina Faso, Sierra Leona y Liberia. Además del terrorismo, los autores destacan como herencia de un Estado corrupto las tensiones sectarias y las luchas tribales (Martinez-Vazquez et al., 2007).

Algunas iniciativas científicas también se preocuparon por investigar formas de mitigar las crisis de inseguridad, probando que empresas y gobiernos deberían entender que aumentando la seguridad en los países, aumentarían la confianza en la realización de negocios internacionales (Efobi et al., 2015). El efecto de la falta de inversión internacional causa un efecto de incremento del terrorismo y de la corrupción. Ese fenómeno fue observado en Nigeria cuando el gobierno local se involucró en corrupción endémica, aliada a un coctel de mala gobernanza, con fallas generalizadas en las políticas estatales, pobreza y desempleo (Uzodike y Maiangwa, 2012).

En los ańos 2000, la academia vio que sus investigadores sociales se concentraron en estudios centrados en el círculo vicioso de la corrupción-terrorismo. La máxima proferida por el ex presidente norteamericano, George Bush, nunca estuvo tan de moda: "La pobreza no transforma personas pobres en terroristas y asesinos". Entretanto, pobreza, corrupción y represión representan una combinación tóxica en muchas sociedades, llevando a gobiernos débiles a la incapacidad 
de hacer cumplir el orden o patrullar sus fronteras, y que son vulnerables a redes terroristas y carteles de drogas (Bush, 2002). La escala del terror percibida en el inicio de los años 2000 trajo evidencias contundentes de que la corrupción actúa como uno de los principales elementos estructurales para el terrorismo (Cramer y Krueger, 2016).

\section{METODOLOGÍA}

Los siguientes análisis tuvieron como objetivo crear clústeres para investigaciones relacionadas con la expresión administrative corruption. Las búsquedas se realizaron junto al banco de datos e investigaciones efectuadas por los usuarios de Google Search.

\section{Procedimientos específicos}

La expresión administrative corruption fue el tema de una búsqueda en las siguientes bases de datos: Direct Science, OneFile (Gale), Web of Science, Taylor \& Francis Online, JsTor Archival Journals, Sage Journals Publications, Directory of Open Access Journals, Emerald Insight, Springer, Technology Research Database, Materials Science \& Engineering Database, y Advanced Technologies \& Aerospace Database. Se analizaron títulos, palabras clave y resúmenes.

Los resúmenes, ańos, autores y títulos de los periódicos fueron incluidos en una planilla y categorizados según los siguientes criterios: administración pública, corrupción, corrupción administrativa, fraude contable, governance indicators, medio ambiente, mercado financiero, political theory, política, política anticorrupción. Tales criterios fueron seleccionados a partir del sitio Transparency International.

De acuerdo con el sitio Transparency International, fuente principal para las diez categorías desarrolladas en esta investigación, la corrupción puede asumir varias formas e incluir comportamientos como funcionarios públicos exigiendo o recibiendo dinero o favores a cambio de servicios, políticos abusando del dinero público o concediendo empleos o contratos públicos a sus patrocinadores, amigos y familiares, y empresas que sobornan a funcionarios para obtener negocios lucrativos. Esta corrupción también puede suceder en cualquier situación: en los negocios, en el gobierno, en los tribunales, en los medios y en la sociedad civil, en la salud y educación, infraestructura y deportes. La corrupción puede involucrar a cualquier persona: políticos, funcionarios del gobierno, funcionarios públicos, empresarios o miembros del público. La corrupción se ajusta a diferentes contextos y circunstancias en mudanza. Puede evolucionar en respuesta 
a mudanzas en las reglas, legislación y hasta tecnología, y genera cuatro tipos de costos: 1) costos económicos, 2) políticos, 3) sociales y 4) ambientales (Transparency International, 2020).

A partir de tales suposiciones, los 253 artículos relacionados con la cA fueron analizados a partir de los algoritmos para clúster y análisis de correspondencia.

\section{Técnica de análisis de datos}

Los análisis que se realizaron en esta investigación se basan en tres técnicas: 1) análisis de clúster, realizado con el soporte del software VOSviewer ${ }^{\circledR}$; 2) análisis de correspondencia, realizado a partir del sistema Sphinx léxica ${ }^{\circledR}$ y 3 ) análisis de incidencias, realizado con la función Trends do Google ${ }^{\oplus}$.

Cabe resaltar que la operación con análisis factorial de correspondencia distribuye en uno de los planos espaciales elementos que, cuanto más distantes, presentan menos relación entre sí. Es importante observar que la disposición de agrupamientos en polos opuestos en el plano de los ejes no necesariamente indica relación de oposición semántica de esos mismos agrupamientos. Como resultado, el Sphinx ${ }^{\oplus}$ ofrece dos ejes, con las disposiciones de las formas reducidas y clases en el plano y la información sobre cuál de los ejes compone de manera más fuerte la disposición de los elementos. A partir de este resultado y de la lectura de los resultados presentados anteriormente, es posible para el investigador nombrar los ejes y sus respectivos polos.

\section{ANÁLISIS DE LOS DATOS}

El análisis de datos fue realizado previendo que el terrorismo y la corrupción relacionada están en crecimiento significativo después del incremento de la lucha (post 11 de septiembre de 2001) contra los grupos extremistas.

Cuando se analiza el trío corrupción, terrorismo y religión es muy importante tener en mente que el volumen de violencia que ocasionan esos factores aumentó en las últimas dos décadas. A partir de enero de 1998, cuando los primeros datos comparables están disponibles, el mundo sufrió aproximadamente 4800 muertes por ataques terroristas. En los seis años siguientes a los ataques en Estados Unidos, a partir de octubre de 2001, terroristas mataron cerca de 11800 personas en todo el mundo. Ese número excluye fatalidades resultantes de ataques terroristas en los conflictos activos en Irak y Afganistán (Berman, 2011).

Los análisis son encaminados a partir del análisis descriptivo de las publicaciones, análisis de clúster, análisis de correspondencia y análisis de la relación entre corrupción y terrorismo. 
CUADRO 1. Categorías relacionadas con la corrupción

\begin{tabular}{lcc}
\hline Tema & Frecuencia & Porcentaje \\
\hline Administración pública & 23 & 18.3 \\
Política anticorrupción & 22 & 17.5 \\
Corrupción administrativa & 17 & 13.5 \\
Governance Indicators & 15 & 11.9 \\
Political Theory & 15 & 11.9 \\
Corrupción & 15 & 11.9 \\
Mercado financiero & 12 & 9.5 \\
Fraude contable & 3 & 2.4 \\
Política & 2 & 1.6 \\
Medio ambiente & 2 & 1.6 \\
Total & 126 & 100.0 \\
\hline
\end{tabular}

Fuente: Elaboración propia.

El interés por el tema "corrupción administrativa" es creciente en el área de negocios. Los primeros registros datan de 1964. Hasta 2019, 253 artículos científicos con evaluación en el sistema double review fueron publicados.

El análisis preliminar buscó crear diez temas a partir de bibliografía relacionada (Caiden, 2001; Caiden y Caiden, 1977; Cerqueti y Coppier, 2016; De Asis, 2001; Jiménez y García, 2018; Taryn Vian, 2020; Werner, 1983). Los tres temas más importantes investigados fueron: administración pública (18.30\%), política anticorrupción (17.50\%) y corrupción administrativa (13.50\%), como se puede observar en el cuadro 1.

Algunas expresiones se relacionaron de forma significativa con las categorías creadas, como fue el caso de administración pública y corrupción; corrupción y política; corruption y countries. Los nodos creados entre las categorías y las palabras colocan en evidencia las asociaciones más fuertes. Generalmente involucran la palabra corruption.

Considerando el periodo de 1964 a 2020, fueron publicados 253 artículos. Los temas que fueron investigados con mayor incidencia fueron corrupción (21.74\%), administración pública (19.37\%) y corrupción administrativa (16.21\%). Cabe resaltar que el tema política anticorrupción surgió después de 2001, cuando el tema fue investigado en 14.23 por ciento de los casos indicados. 
ESQUEMA 1. Categorías y puntos importantes

\begin{tabular}{|c|c|c|c|}
\hline Administración pública [130] & Corruption [35] & Political [17] & Administrative [14] \\
\hline Corruption [116] & Corruption [28] & Political [17] & Countries [12] \\
\hline Polanticorrupt [114] & Corruption [22] & Political [17] & Countries [09] \\
\hline Adm Corrupt [111] & Corruption [25] & Political [16] & Governance [10] \\
\hline Political Theo [80] & Corruption [22] & Administrative [09] & Countries [08] \\
\hline Indicators [51] & Corruption [14] & Political [07] & Governance [06] \\
\hline Mercado financiero [38] & Corruption [08] & Political [07] & Governance [04] \\
\hline Medio ambiente [11] & Corruption [08] & Political [01] & Reform [01] \\
\hline Fraude [5] & Corruption [02] & Political [02] & Governance [01] \\
\hline Política [05] & Corruption [02] & Political [01] & Administrative [01] \\
\hline
\end{tabular}

Fuente: Elaboración propia.

A partir de 2001, el tema gana atención, materializada en la cantidad de publicaciones científicas. De 1964 a 2000, se habían publicado apenas 24 artículos científicos centrados en corrupción administrativa (9.4\%). El año de 2001 se presenta como una piedra angular para el tema corrupción. Dos posibles explicaciones para ese interés repentino son el escándalo del fraude contable aplicado por la Arthur Andersen junto a Enron (octubre de 2001) y los atentados realizados por la AlQaeda a las instalaciones norteamericanas (septiembre de 2001). Ese ataque fundamentalista del 11 de septiembre creó una estrecha y fuerte relación entre el terrorismo $y$ el financiamiento del terror (FBI, 2002).

Después del análisis del aumento de las publicaciones, es posible crear un filtro que envuelva solo las diez categorías principales extraídas del sitio TI.

El tema centrado en las diez categorías también tiene interés creciente a partir de 2001 (gráfica 1). La línea azul (rombo) muestra que las categorías creadas para corrupción administrativa acompañan el tema general de las 253 publicaciones.

A partir de la gráfica 2 es posible percibir que tales publicaciones aún no forman una escuela de pensamiento o red de investigación mundial. A pesar de 
GRÁFICA 1. Evolución del tema corrupción administrativa

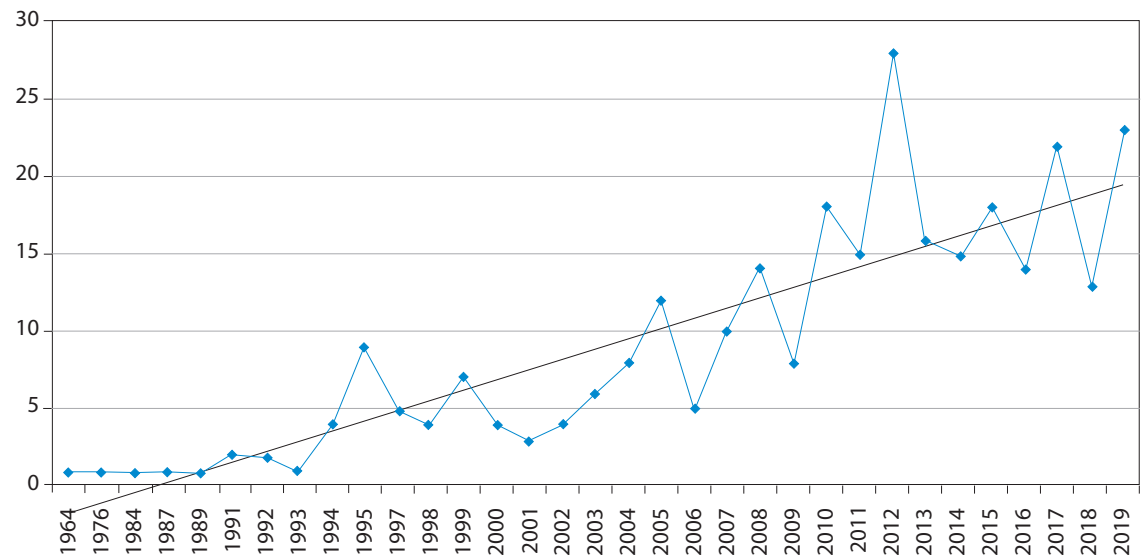

Fuente: Elaboración propia.

GRÁFICA 2. Categorías relacionadas con la corrupción y la corrupción administrativa

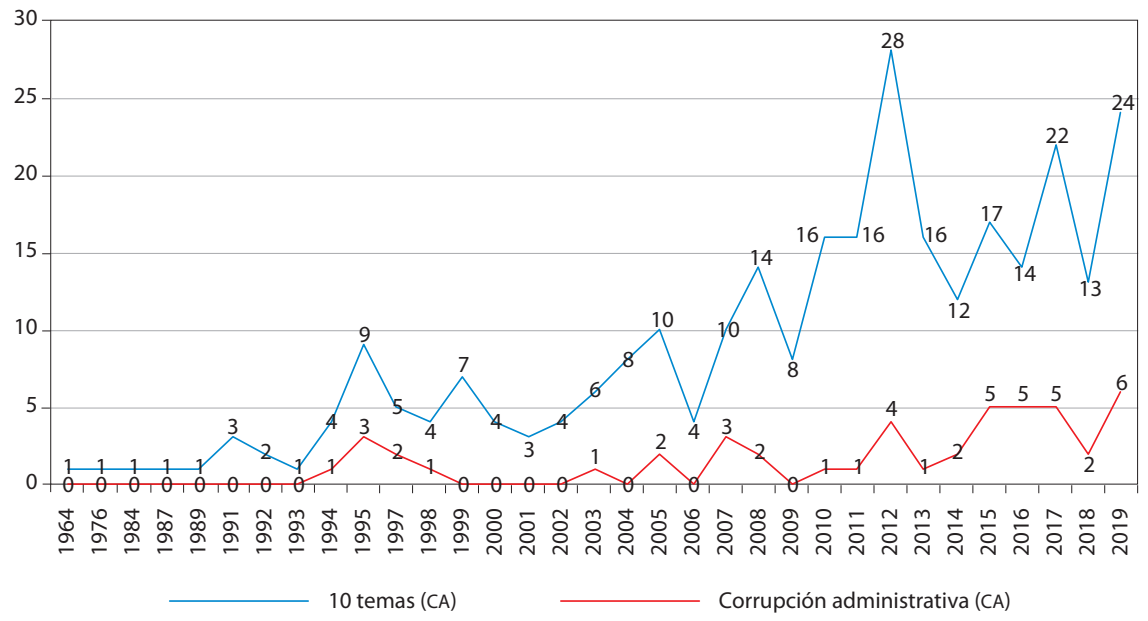

Fuente: Elaboración propia. 
ESQUEMA 2. Clústeres administrative corruption

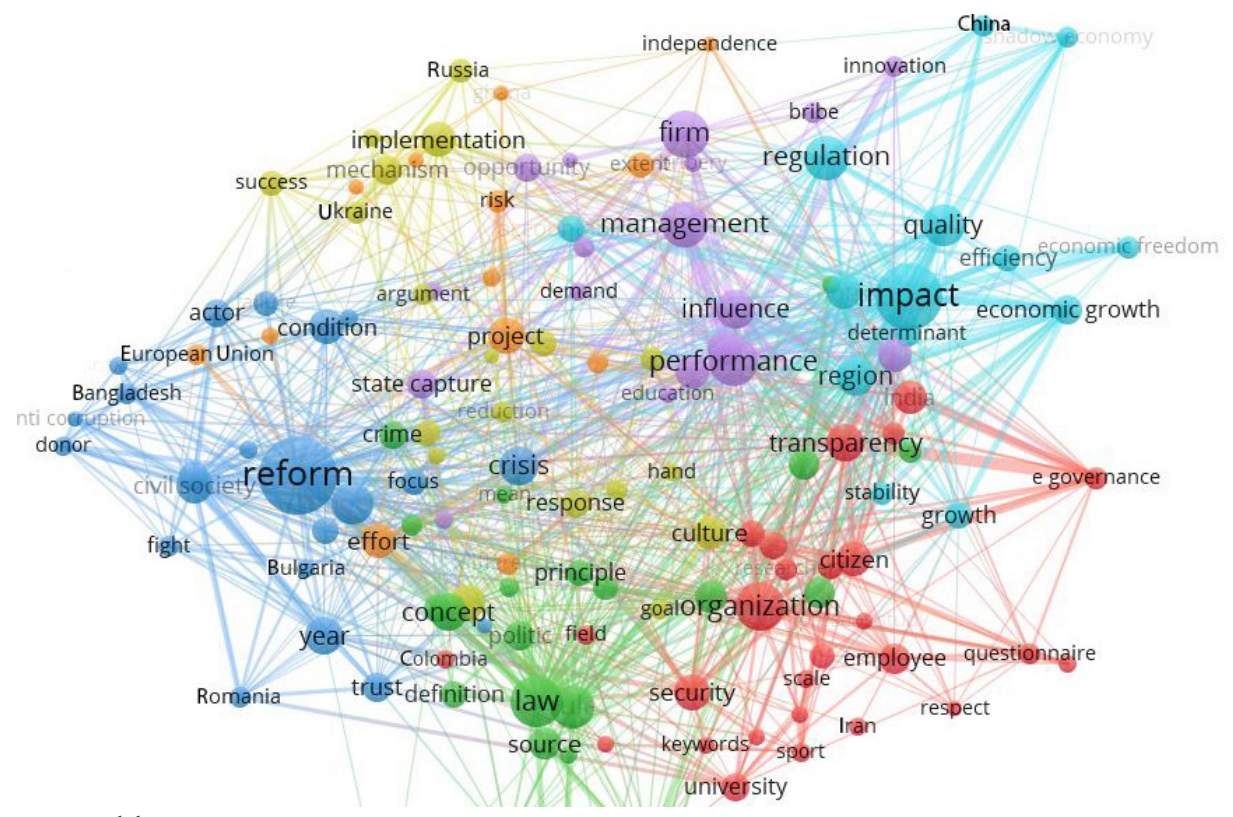

Fuente: Elaboración propia

creciente, ese interés no sigue una línea constante y ni posee una red de investigadores involucrados con el asunto, aspecto evidenciado también en el análisis realizado en el VOSviewer ${ }^{\circ}$. Esta evaluación fue realizada con la selección de tres expresiones: investigación científica administrative corruption, investigación de transparencia y corrupción administrative corruption y la búsqueda por el tema administrative corruption. En el esquema 2 se ilustran esas relaciones y los cinco agrupamientos.

El clúster 1 reúne las investigaciones que tienen como objetivo entender las reformas administrativas realizadas por los Estados y su conexión con las crisis económicas. La interacción del clúster 1 con los agrupamientos 2 y 3 , que tratan sobre la gestión y las leyes sobre corrupción administrativa, es fuerte.

El clúster 2 evalúa las leyes anticorrupción, principios, definiciones y política. Este clúster interactúa de forma estrecha con los clústeres 1 y 5 .

El clúster 3 analiza la actuación de las empresas con la corrupción, así como la gestión, las oportunidades y la innovación. Este clúster está incluido entre los clústeres 1 y 4. 
El clúster 4 trata de un agrupamiento que mide los impactos de las políticas anticorrupción en el universo de los negocios. Temas como reglas, calidad y eficiencia son objetivos frecuentes para ese agrupamiento en el universo de la investigación sobre corrupción administrativa. El clúster 4 actúa de forma estrecha con los agrupamientos 3 y 5 .

El clúster 5 reúne los intereses en investigación sobre la metodología de la investigación científica en corrupción administrativa. Son subtemas comunes a ese clúster cuestiones como cultura, empleo de cuestionarios, muestreo y laboratorios de estudio. Son clústeres próximos los de número 2 y 4.

El conjunto de cinco clústeres, por lo tanto, investiga gestión, leyes, acciones, transparencia y eficiencia de las prácticas e investigaciones académicas. Algunas oportunidades pueden nacer de las interacciones entre los agrupamientos, tales como los espacios entre las reformas administrativas, leyes y desempeño o, incluso, entre reformas e impactos de las acciones anticorrupción. Cuando tales agrupamientos se relacionan con el trabajo de Transparency International es posible identificar nuevos clústeres. Sería posible crear otros ocho clústeres e iniciar nuevas investigaciones. Esos agrupamientos serían: a) acceso a la información;

Cuadro 2. Cuadro resumen

\begin{aligned} & Clústeres categóricos y Transparency International \\ & \hline 1 Reformas administrativas \\ & 3 Leyes y principios anticorrupción \\ & 4 Desempeño-oportunidades \\ & 5 Impactos de las políticas anticorrupción \\ & 6 Metodología de la investigación científica \\ & 7 Acceso a la información \\ & 8 Accountability \\ & 9 Recuperación de activos \\ & 10 Auditoría \\ & 11 Cambio de informaciones \\ & 12 Fraude \\ & 13 Secreto de propiedad \\ & 14 Soborno \\ & \hline\end{aligned}

Fuente: Transparency International (2020). 
b) accountability; c) recuperación de activos; d) auditoría; e) cambio automático de informaciones; $f$ ) fraude en la transferencia de lucros; $g$ ) secreto de propriedad y h) soborno.

El análisis permitió identificar las tendencias, cómo están trabajando los grupos de investigación, oportunidades y estructuras en la investigación sobre corrupción administrativa. Sin embargo, el análisis no evidencia lo que los individuos están investigando en internet. $\mathrm{O}$ sea, se cuestiona el hecho de existir una brecha entre las investigaciones científicas y el interés de las personas por el tema.

La gráfica 3 muestra que las personas están interesadas en maneras de enfrentar la corrupción y el soborno. Esa sería la mayor preocupación constatada. Tales evidencias surgieron a partir del empleo del sistema Google Trends ${ }^{\odot}$. Los países que lideran la búsqueda por el tema administrative corruption son Burkina Faso (85/180), Zimbabue (158/180), Etiopía (96/180), Zambia (113/180) y Uganda (137/180). Los valores entre paréntesis indican cómo están posicionados los países en el ranking mundial de corrupción (ranking de 180 puestos) (Transparency International, 2020). Es perceptible que países con elevados valores para el ranking de corrupción mundial tengan habitantes preocupados por el tema y por la forma de combatir la corrupción administrativa.

Tales países no son los más corruptos del mundo, son las naciones donde ocurre la mayor parte de las investigaciones sobre corrupción en el mundo. Las

GRÁFICA 3. Corrupción y terrorismo

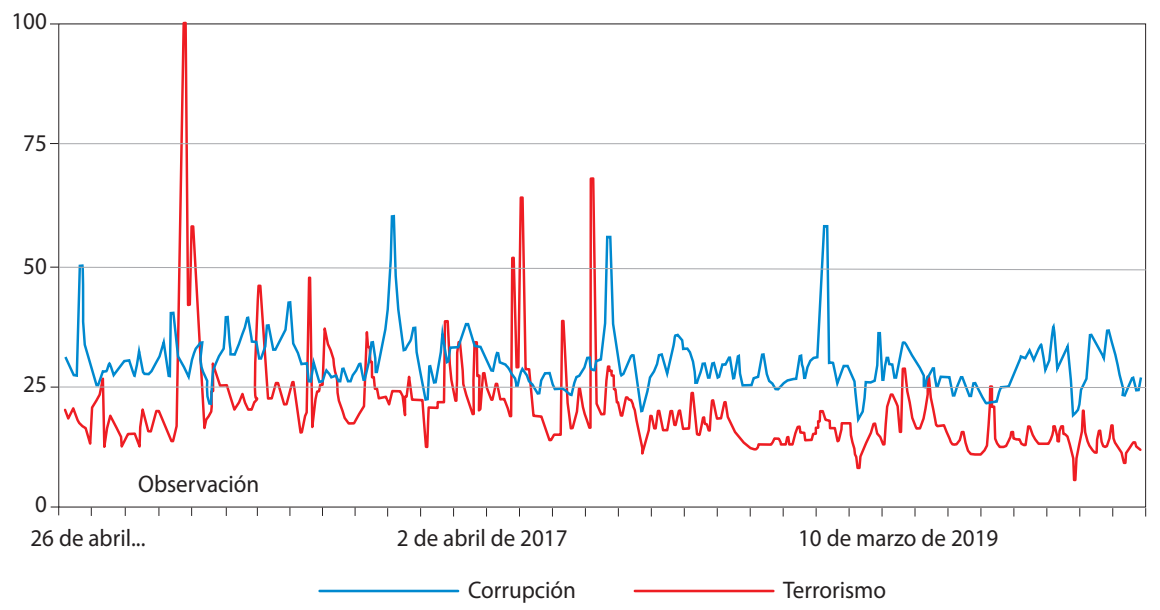

Fuente: Elaboración propia. 
ESQUEMA 3. Clústeres y palabras más importantes

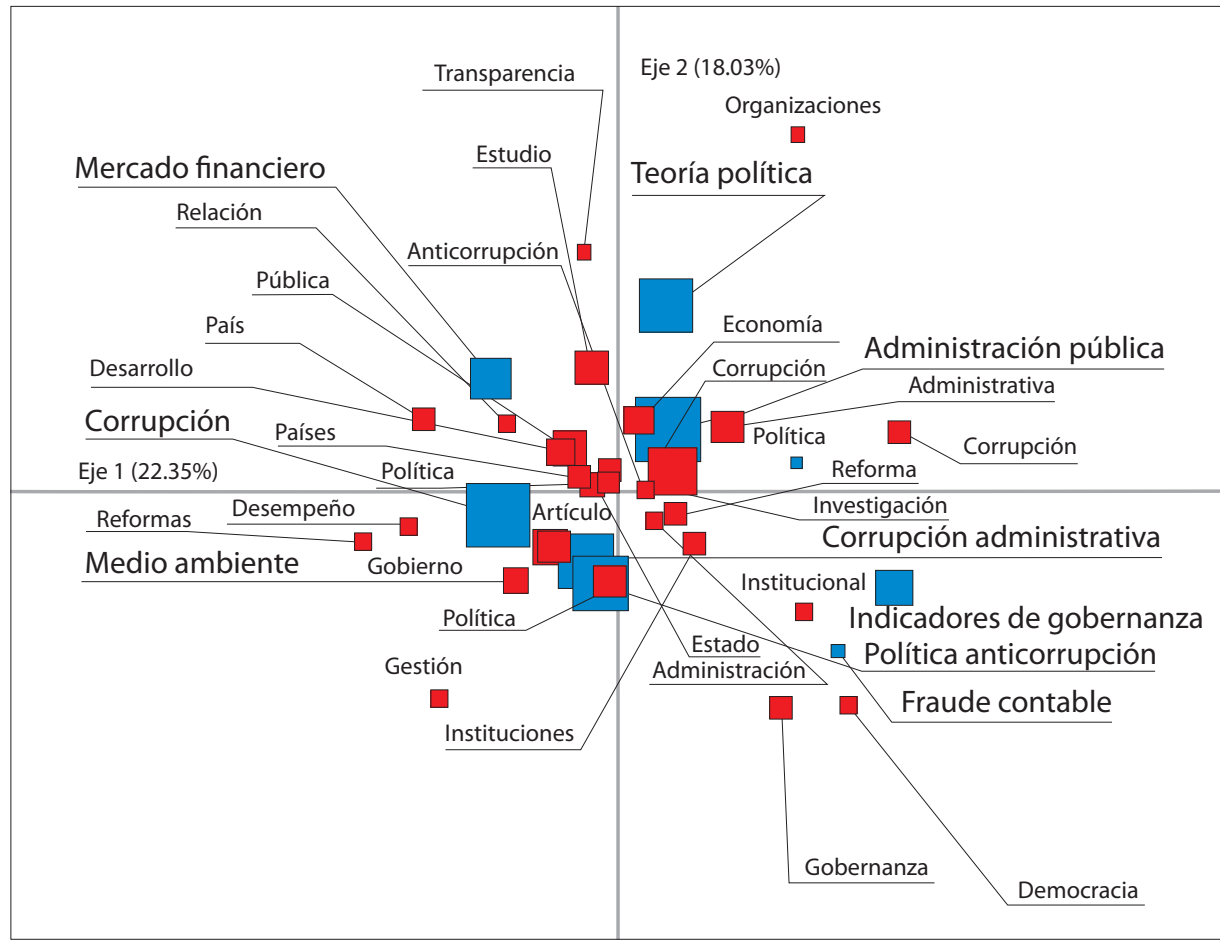

Fuente: Elaboración propia.

expresiones relacionadas con más frecuencia a administrative corruption son "terrorismo" y "corrupción" (gráfica 3). Se observa que en el periodo de 2004 a 2020 la búsqueda por terrorismo y corrupción estuvo intensamente relacionada.

El análisis de correspondencia fue creado a partir del Sistema Sphinx Léxica ${ }^{\oplus}$, cuando se buscaron las palabras más citadas e importantes de los 253 artículos publicados de 1964 a 2020. El cruce se realizó entre las palabras más importantes y las diez categorías creadas anteriormente (political theory, medio ambiente, corrupción, administración pública, corrupción administrativa, política anticorrupción, mercado financiero, fraude contable y governance indicators) (Transparency International, 2020). A partir del esquema 3 es posible identificar cuatro cuadrantes para el análisis: 1) mercado financiero; 2) political theory y administración pública; 3) medio ambiente y corrupción y 4) governance indicators y fraude. 
El cuadrante 1 indica una relación entre el mercado financiero e investigación, desarrollo y estudios anticorrupción. Ese cuadrante puede ser percibido como el cuadrante de la investigación de la corrupción administrativa, asociado de forma significativa con el estudio del mercado financiero. La palabra más representativa para el cuadrante 1 es "estudio".

El cuadrante 2 sugiere una relación importante entre administración pública, economía y corrupción. Esa relación se presenta nuevamente de forma significativa cuando estudios sobre terrorismo y corrupción son relacionados (Cramer y Krueger, 2016; Poole-Robb y Bailey, 2002).

El cuadrante 3 apunta hacia un agrupamiento construido a partir del medio ambiente, gobierno e instituciones privadas. Este cuadrante muestra una relación entre el gobierno y las empresas privadas en el sentido de obtener ventajas en algún favorecimiento ambiental.

El cuadrante 4 presenta los indicadores de fraude contable, de gobernanza y de democracia como un sistema y una estrategia de lucha contra la corrupción administrativa.

Cuando todos los análisis son confrontados, se observa que hay un descompás entre la problemática CA, y los intereses de investigación no entran en alineamiento. Hay también oportunidades entre clústeres, como, por ejemplo el cuadrante 1 y el cuadrante 3 , que unidos pueden apuntar una problemática: corrupción y sustentabilidad (mercado financiero vs medio ambiente). Análisis como este pueden extenderse a la unión de otros clústeres del análisis de correspondencia.

Una de las primeras explicaciones para ese descompás es la cuestión de la amplitud de las investigaciones. Las investigaciones acerca de la corrupción administrativa contemplan el panorama general y no especificidades (Haghighatian et al., 2013). Tales especificidades podrían ser cuestiones como la ausencia de disciplinas éticas que son ignoradas en la escuela, cuando los fundamentos de los valores deberían trabajarse mejor (Cortés, 2020; Muñoz Estrada, 2015).

Puntos de subjetividad comportamentales también fueron ignorados en las investigaciones, pero emergen de los datos como oportunidades para investigaciones relacionadas con la CA. Por ejemplo, el estado de bienestar del individuo frente a los actos de corrupción (Sulemana et al., 2017) y el desarrollo del espíritu emprendedor como disciplina de ética y de la virtud (Al Baghlani, 2018; Badulescu y Badulescu, 2012; Stanfill et al., 2016; Williams, 2014; Williams et al., 2013). Otro tema con oportunidad de publicación es el "perjuicio monetario" relacionado con la "corrupción administrativa" (Del Castillo et al., 2019; Lemma, 2015); fueron pocos los trabajos publicados con ese tema. 
Cabe resaltar también algunas iniciativas que se posicionaron al margen de todos los clústeres: la simulación en juegos (experimentación y simulaciones en laboratorio) como práctica para identificar aspectos de la CA. Algunas de esas investigaciones simularon ambientes de licitaciones y contratos de contratistas y estímulos eran enviados para el entendimiento de cómo se manifiesta la corrupción entre los participantes (Antoci y Sacco, 1995). Hubo también casos de investigaciones que simularon la punición por corrupción a partir de bancos de datos (Carlin et al., 2015).

La omisión en investigación también podría entenderse en el fenómeno "retirar la escalera”, un término creado por HaJoon Chang, que se refiere a los países desarrollados que inhiben el acceso al desarrollo para los países pobres, aspecto determinante, según los autores, para la presencia de la corrupción administrativa (Ballesteros, 2016; Hanlon, 2004). La cuestión sugiere otras brechas de publicación, como un círculo vicioso apuntado en dos estudios.

La burocracia aliada al sistema judiciario (Mauro, 1995) y gobiernos débiles con elevada tributación fomentan prácticas de ca (Shleifer y Vishny, 1993). Se deduce que el exceso de fases en los procesos, que confieren conclusiones costosas a los procesos, son estrategias adoptadas por los gobiernos corruptos. A propósito, tales aspectos son difíciles de monitorear y aún hay poca investigación publicada sobre el tema.

La escasez de estudios en el área aumenta cuando este punto se relaciona con políticas públicas mal ejecutadas. Ese tipo de corrupción puede tener las consecuencias más graves para una sociedad. Situaciones extremas de ese tipo de corrupción, como la de un dictador que no distingue entre su propia riqueza y la del país o toma decisiones políticas que atienden exclusivamente a sus propios intereses, son un poco más fáciles de estudiar (Jain, 2001).

Las brechas también están presentes en otros puntos. Las llamadas externalidades se hacen presentes de las más variadas maneras (Senci, 2019), lo que contribuye a la falta de entendimiento sobre la corrupción. Un ejemplo de externalidad con la corrupción ocurrió en Italia, cuando el aumento de prisiones de traficantes hizo que la corrupción aumentase en la misma proporción (20\%). Las facciones criminales operaban en el interior de las prisiones, utilizando la seguridad de la cárcel para las operaciones de narcotráfico y corrupción en general. En este periodo hubo un aumento del malestar social y un consenso en favor de una justicia criminal más rigurosa (Melossi y Pavarini, 2018). Una de las posibles explicaciones para este fenómeno puede ser la falta de sincronismo entre el poder público y la sociedad en el combate a la corrupción, tal como se previó en algunos estudios (Pillay, 2004). 
Otra cuestión surge del análisis de los datos y se presenta en estudios que retratan las grandes desigualdades provocadas por gobiernos corruptos y el poder económico: el incremento de la corrupción durante las grandes crisis económicas, que a su vez incrementa las crisis humanitarias causando grandes catástrofes (Avilés, 2019). Es recurrente que tales crisis humanitarias, provocadas por un colapso democrático, disminuyan cuando las democracias tienen una burocracia de mayor calidad (Krishnarajan, 2019). El exceso de trámites en los procesos administrativos es visto como una herramienta que favorece la CA.

El análisis de correspondencia, en por lo menos dos cuadrantes, también sugiere algunos puntos de estudio, vistos como oportunidades. Se trata de corrupciones disimuladas como favores o actividades corruptas que traen pérdidas oscuras. Serían ejemplos de tales pérdidas relacionadas con la corrupción disimulada: el aumento de alquileres percibidos en algunas partes del mundo, como ocurrió en India y Turquía (Rose-Ackerman, 1997); apoyos en elecciones presidenciales, mercados negros, importaciones y exportaciones fraudulentas, "underground economies", y lavado de dinero (Kunikov y Rose-Ackerman, 2005; Rose-Ackerman, 1978, 1997, 1999).

\section{CONCLUSIONES}

La búsqueda de soluciones para inhibir la corrupción es el terrorismo creciente, porque ambos problemas caminan juntos y están en amplio crecimiento. Las acciones posteriores al 11 de septiembre ampliaron la violencia causada por ataques terroristas. La taza de muertes por actos terroristas saltó de $109 \mathrm{al}$ mes a $158 \mathrm{al}$ mes. Si los números provenientes de Irak y Afganistán fuesen registrados, el número sería de 529 personas muertas mensualmente en ataques terroristas (Berman, 2011). Ese punto es relevante en este estudio porque se observó que la investigación está desconectada del interés público, la academia estudia corrupción administrativa de forma aislada del terrorismo. Ya fue relacionado en el referencial teórico que ambos forman parte del mismo sistema.

En este estudio se buscó trabajar clústeres para el tema "corrupción administrativa”. A partir de los análisis realizados se constató una serie de evidencias, oportunidades y omisiones en investigaciones. También se realizó un contraste entre la visión de la academia y la demanda de los interesados en el tema CA.

Los investigadores buscan entender la corrupción administrativa evaluando burocracia, antropología, rendición de cuentas, política y economía; las personas buscan entender la corrupción administrativa en conjunto con el crimen organizado, terrorismo y el soborno; Transparency International trata de entender la corrupción admi- 
nistrativa a partir del acceso a la información, accountability, fraude, secreto de propriedad y soborno. Cuando piensan en corrupción, los investigadores no contemplan en sus estudios la cuestión del secreto de propriedad; Transparency International no dedica la debida atención al terrorismo internacional, y las personas son restrictas al soborno cuando quieren entender el tema en cuestión.

Se encontraron oportunidades de investigación sobre el comportamiento de los individuos, realizado en laboratorios o en la vida cotidiana de las personas, algo próximo a los preceptos ya desarrollados por la economía comportamental (Gino y Ariely, 2012; Mazar y Ariely, 2008). Es posible también identificar investigaciones convenientes sobre la sustentabilidad vs corrupción administrativa y corrupción $v$ s crimen organizado y terrorismo.

Los análisis también apuntaron regiones centrales en los mapas, que deben ser observadas como puntos de saturación en la temática de ca. Son regiones de exceso de ofertas en virtud de la cantidad de publicaciones que se concentran en ella. En este punto se destacan investigaciones políticas, teorías políticas, prácticas anticorrupción, reformas administrativas y sus impactos, así como el estudio de las leyes anticorrupción.

A partir del análisis de contenido realizado en los abstracts fue posible entender qué estudios de Data Science en análisis de ca no se están realizando. Si se entiende que la CA se relaciona con el concepto del hombre "biológicamente cultural" (Pinker, 2009), hay bastantes oportunidades por explorar en el análisis de grandes bancos de datos o investigaciones experimentales. También se observó poca atención a la creación de una representatividad en las muestras, omisión importante cuando ya se demostró que la corrupción opera en redes en un universo de economía global; las descubiertas en esta investigación sugieren que la corrupción también es impulsada por la codicia o el interés propio.

Ese efecto también se ha observado en estudios recientes que han evidenciado que la principal motivación para el soborno es la reciprocidad obtenida en un ambiente de codicia. Las investigaciones podrían entonces concentrarse en la prevención de sobornos que dependen de un determinado resultado. Y si la reciprocidad motiva el soborno, las intervenciones políticas deben concentrarse en tornar la reciprocidad más difícil, por ejemplo, disminuyendo el contacto personal a través del anonimato y la rotación de personal (Gneezy et al., 2019).

Tal vez la contribución más importante de esta investigación esté relacionada con las externalidades provocadas por la corrupción. Ya se relato el efecto en el valor de los alquileres de inmuebles en regiones permeadas por la corrupción y las fugas de inversiones en determinados países, pero ¿qué más podría ser relacio- 
nado como efectos externos positivos o negativos para la temática corrupción administrativa?

La evaluación de clúster realizada abre una posibilidad de agenda futura para investigaciones sobre la corrupción administrativa, cuando sugiere: $a$ ) alineamiento entre las investigaciones; b) nuevas investigaciones que contemplen los tres análisis, y $c$ ) una investigación que busque entender por qué hay esa diferencia entre lo que se investiga y lo que se demanda investigar. GEิ

\section{REFERENCIAS}

Afzali, A.R. (2011), "Administrative Corruption and its Impact on Development: Causes, Consequences and Exit Strategies", Journal of International Law, 45, pp. 235-264.

Ajayi, O. y G.H. Millard (1997), "Drugs and Corruption in Latin America", Dickinson Journal of International Law, 15(3), pp. 543-533.

Al-Jundi, S.A. (2019), "A Survey Dataset on Determinants of Administrative Corruption", Data in Brief, 27, Dor: https://doi.org/10.1016/j.dib.2019.104820.

Al-jundi, S., A.Schuhaiber y S. Alemara (2019), "Why is Administrative Corruption Pervasive? A Mediation Approach", Journal of Applied Economic Sciences, 14(1), pp. 178-194, DOI: https://doi.org/10.14505/jaes.v14.1(63).17.

Al Baghlani, A.L.K. (2018), "An Investigation into Strategies Used by Iraqi Smes to Survive in the hostile Environment: The Case of Al-Khaleej Company", Academy of Entrepreneurship Journal, 24(1), p. 1.

Angel Olofinbiyi, S. (2020), "The Ideological Basis of Boko Haram Terrorism : A Review Essay", African Journal of Terrorism and Insurgency Research, S1(1), pp. 141-154, DOI: https://doi.org/10.31920/2732-5008/2020/S1n1a8.

Antoci, A. y P.L. Sacco (1995), "A Public Contracting Evolutionary Game with Corruption", Journal of Economics Zeitschrift Für Nationalökonomie, 61(2), pp. 89-122, DoI: https:// doi.org/10.1007/BF01238776.

Avilés, W. (2019), "The Wayúu Tragedy: Death, Water and the Imperatives of Global Capitalism”, Third World Quarterly, 40(9), pp. 1750-1766, Dor: https://doi.org/10.1080 /01436597.2019.1613638.

Badulescu, D. y A. Badulescu (2012), "Corruption as Constraint in SMEs Financial Management (I- Concept and Economic Impact)", Journal of Electrical and Electronics Engineering, 5(1), pp. 7-12.

Ballesteros, I.E.S. (2016), "Corrupción y desafíos organizacionales en un mundo de asociaciones público-privadas”, Gestión y Política Pública, XXV(2), pp. 365-413.

Becker, G. (1968), “Crime and Punishment: An Economic Approach”, Journal of Political Economy, 76(2), pp. 169-217. 
Berman, E. (2011), Radical, Religious, and Violent The New Economics of Terrorism, Cambridge, The міт Press.

Bertock, J. (2001), "Construcción de una infraestructura de la ética: Las experiencias recientes de los países de la ocDE", Gestión y Política Pública, X(2), pp. 335-373.

Boussiga, N. y M. Ghdamsi (2016), "The Corruption-Terrorism Nexus: A Panel Data Approach", International Journal of Economics and Finance, 8(11), DoI: https://doi. org/10.5539/ijef.v8n11p111.

Bush, G.W. (2002), "Securing Freedom's Triumph”, New York Times, 11 de septiembre.

Caiden, G. (2001), "Dealing with Administrative Corruption”, en T.L. Cooper (ed.), Handbook of Administrative Ethics, 2a. ed., Nueva York, Marcel Dekker.

Caiden, G. y N. Caiden (1977), "Administrative Corruption”, Public Administration Review, 37(3), pp. 301-309.

Carlin, R., G. Love y C. Martínez-Gallardo (2015), "Cushioning the Fall: Scandals, Economic Conditions, and Executive Approval”, Political Behavior, 37(1), pp. 109-130, DoI: https://doi.org/10.1007/s11109-014-9267-3.

Cerqueti, R. y R. Coppier (2016), "Corruption, Evasion and Environmental Policy: A Game Theory Approach”, IMA Journal of Management Mathematics, 27(2), pp. 235-253, DoI: https://doi.org/10.1093/imaman/dpu019.

Clutterbuck, R. (1995), "Peru: Cocaine, Terrorism and Corruption", International Relations, 12(5), pp. 77-92, Dor: https://doi.org/10.1177/004711789501200506.

Cortés, G.P. (2020), “Un modelo de choques e interrupciones de reforma anticorrupción”, Gestión y Politica Pública, XXIX(2), pp. 295-319.

Cramer, J. y A.B. Krueger (2016), "Disruptive Change in the Taxi Business: The Case of Uber”, American Economic Review, 106(5), pp. 177-182, Dor: https://doi.org/10.1257/ aer.p20161002.

Crenshaw, M. (1981), “The Causes of Terrorism”, Comparative Politics, 13(4), pp. 379-399, Dor: https://doi.org/10.2307/421717.

de Asis, M.G. (2001), "Construcción de coaliciones para luchar contra la corrupción”, Gestión y Política Pública, X(2), pp. 309-319.

del Castillo, A. (2001), "El soborno: Un marco conceptual para su análisis", Gestión y Politica Pública, X(2), pp. 276-307.

Del Castillo Alemán, G. y G. Flores Ivich (2019), "La cobertura de la reforma educativa en México: Un asunto de medios y políticas”, Gestión y Política Pública, XXVIII(2), pp. 501-539, Dor: https://doi.org/10.29265/gypp.v28i2.628.

Efobi, U., S.A. Asongu y I. Beecroft (2015), "Foreign Direct Investment, Aid and Terrorism: Empirical Insight Conditioned on Corruption Control”, SSRN Electronic Journal, pp. 1-23, DoI: https://doi.org/10.2139/ssrn.2586355. 
Esoimeme, E.E. (2020), "Identifying and Reducing the Money Laundering Risks Posed by Individuals Who Have Been Unknowingly Recruited As Money Rules", Journal of Money Laundering Control, 24(1), pp. 201-212, DoI: https://doi.org/10.1108/JMLC05-2020-0053.

Factor, R. y M. Kang (2015), "Corruption and Population health Outcomes: An Analysis of Data from 133 Countries Using Structural Equation Modeling”, International Journal of Public Health, 60(6), pp. 633-641, Dor: https://doi.org/10.1007/s00038-015-0687-6. FBI (Federal Bureau of Investigation) (2002), Financing Patterns Associated with Al Qaeda and Global Terrorist Networks, disponible en: https://archives.fbi.gov/archives/news/ testimony/financing-patterns-associated-with-al-qaeda-and-global-terrorist-networks [fecha de consulta: 20 de abril de 2020].

Gächter, S. y J.F. Schulz (2016), "Intrinsic Honesty and the Prevalence of Rule Violations across Societies”, Nature, 531, pp. 496-499, DoI: https://doi.org/10.1038/nature17160.

Gino, F. y D. Ariely (2012), "The Dark Side of Creativity: Original Thinkers Can Be More Dishonest", Journal of Personality and Social Psychology, 102(3), pp. 445-459, Dor: https://doi.org/10.1037/a0026406.

Gneezy, U., S. Saccardo y R. van Veldhuizen (2019), "Bribery: Behavioral Drivers of Distorted Decisions", Journal of the European Economic Association, 17(3), pp. 917-946, DoI: https://doi.org/10.1093/jeea/jvy043.

Haghighatian, M., S. Karimizadeh y J. Nazari (2013), "An Analysis of Administrative Corruption (Administrative Organizations of Yazd City)", Journal of Applied Sociology, 4(48), pp. 31-34.

Hanlon, J. (2004), "Do Donors Promote Corruption?: The Case of Mozambique", Third World Quarterly, 25(4), pp. 747-763, Dor: https://doi.org/10.1080/01436590410001678960. Hurwitz, A. y R. Huang (2014), Civil War and the Rule of Law: Security, Development, Human Rights, Boulder, Lynne Rienner, Dor: https://doi.org/978-1-58826-531-9.

Jacobs, J.B. y F. Anechiarico (2001), "Sobre los costos y beneficios del control de la corrupción”, Gestión y Politica Pública, X(2), pp. 321-333, disponible en: https://www.redalyc. org/articulo.oa?id=13310207 [fecha de consulta: 20 de abril de 2020].

Jain, A. (2001), "Corruption: A Review", Journal of Economic Surveys, 15(1), pp. 71-121, DoI: https://doi.org/10.1111/1467-6419.00133.

Jancsics, D. y I. Jávor (2012), “Corrupt Governmental Networks", International Public Management Journal, 15(1), pp. 62-99, DoI: https://doi.org/10.1080/10967494.2012 .684019 .

Jiménez, J. y C. García (2018), "Does Local Public Corruption Generate Partisan Effects on Polls? Crime, Law and Social Change, 69(1), pp. 3-23, Dor: https://doi.org/10.1007/ s10611-016-9671-1. 
Krishnarajan, S. (2019), "Economic Crisis, Bureaucratic Quality and Democratic Breakdown", Government and Opposition, 54(4), pp. 715-744, DoI: https://doi.org/10.1017/gov.2017.37. Kunikov, J. y S. Rose-Ackerman (2005), "Electoral Rules and Constitutional Structures as Constraints on Corruption", British Journal of Political Science, 35(4), pp. 573-606, Dor: https://doi.org/10.1017/S0007123405000311.

Lemma, T.T. (2015), "Corruption, Debt Financing and Corporate Ownership", Journal of Economic Studies, 42(3), pp. 433-461, Dor: https://doi.org/10.1108/JES-02-2013-0029.

Magouirk, J. (2008), "The Nefarious Helping Hand: Anti-Corruption Campaigns, Social Service Provision, and Terrorism 1", Terrorism and Political Violence, 20(3), pp. 356-375, Dor: https://doi.org/10.1080/09546550802073318.

Martinez-Vazquez, J., J. Arze del Granado y J. Boex (2007), "Fighting Corruption in the Public Sector", en J. Martinez-Vazquez, J. Arze del Granado y J. Boex (eds.), Contributions to Economic Analysis, vol. 284, Ámsterdam, Elsevier, Dor: https://doi.org/https:// doi.org/10.1016/S0573-8555(06)84001-0.

Mauro, P. (1995), "Corruption and Growth", The Quarterly Journal of Economics, 110(3), pp. 681-712, DoI: https://doi.org/10.2307/2946696.

Mazar, N. y D. Ariely (2008), "Dishonesty in Everyday Life and Its Policy Implications", Journal of Marketing Research, 25(1), pp. 633-644.

Melossi, D. y M. Pavarini (2018), The Prison and the Factory: Origins of the Penitentiary System, Londres, Palgrave Macmillan.

Merari, A. (1991), "Academic Research and Government Policy on Terrorism", Terrorism and Political Violence, 3(1), pp. 88-102, Dor: https://doi.org/10.1080/09546559108427094.

Merari, A. (1993), "Terrorism as a Strategy of Insurgency", Terrorism and Political Violence, 5(4), pp. 213-251.

Mousavi, P. y M. Pourkiani (2013), "Administrative Corruption: Ways of Tackling the Problem", European Online Journal of Natural and Social Sciences, 2(3), pp. 178-187.

Mungiu-Pippidi, A. (2013), "Controlling Corruption Through Collective Action", Journal of Democracy, 24(1), pp. 101-115, Dor: https://doi.org/10.1353/jod.2013.0020.

Mungiu-Pippidi, A. y A.E. Dusu (2011), "Civil Society and Control of Corruption: Assessing Governance of Romanian Public Universities", International Journal of Educational Development, 31(5), pp. 532-546, Dor: https://doi.org/https://doi.org/10.1016/j.ijedudev.2010.03.016.

Muñoz Estrada, E.S. (2015), “Construcción del imaginario sobre la diversidad cultural: Un estudio desde el discurso en los niños y niñas del municipio de Riohacha”, Boletín Redipe, 4(9), pp. 40-51.

Nehemia, F. y A. Merari (1985), "Impact of Terrorism: A Double-edged Sword”, Political Psychology, 1(1), pp. 591-604. 
Pillay, S. (2004), "Corruption - The Challenge to Good Governance: A South African Perspective", International Journal of Public Sector Management, 17(7), pp. 586-605, DOI: https://doi.org/10.1108/09513550410562266.

Pinker, S. (2009), Como a mente funciona, São Paulo, Companhia das Letras.

Poole-Robb, S. y A. Bailey (2002), Risky Business: Corruption, Fraud, Terrorism and other Threats to Global Business, Londres, Kogan Page.

Rose-Ackerman, S. (1978), "Bureaucratic Structure and Corruption", Corruption: A Study in Political Economy, Ámsterdam, Elsevier, pp. 167-188, Dor: https://doi.org/10.1016/ B978-0-12-596350-3.50013-2.

Rose-Ackerman, S. (1997), “The Role of The World Bank in Controlling Corruption”, Law and Policy in International Business, 29(1), pp. 93-114.

Rose-Ackerman, S. (1999), Corruption and Government: Causes, Consequences and Reform. Cambridge, Cambridge University Press.

Santin, J.R. y L. Frizon (2020), "Administração consensual , accountability e transparência na administração pública brasileira", Revista de Direito da Cidade, 12(2), pp. 504-524, DOI: https://doi.org/10.12957/rdc.2020.48608.

Senci, C.M. (2019), "Corruption and Externalities: Assessing the Role of Intentions", Papers: Revista de Sociologia, 105(1), pp. 111-141, Dor: https://doi.org/10.5565/rev/papers.2630.

Shelley, L. (2004), "The Unholy Trinity: Transnational Crime, Corruption, and Terrorism", The Brown Journal of World Affairs, 11(2), pp. 101-111.

Shleifer, A. y R.W. Vishny (1993), "Corruption”, The Quarterly Journal of Economics, 108(3), pp. 599-617, Dor: https://doi.org/10.2307/2118402.

Stanfill, B.A., A.D. Villarreal, M.R. Medina, E.P. Esquivel, E. de la Rosa y P.A. Duncan (2016), "Beyond the Culture of Corruption: Staying Ethical While Doing Business in Latin America", Journal of Organizational Culture, Communications and Conflict, 20(1), pp. 59-80.

Sulemana, I., A.M. Iddrisu y J.E. Kyoore (2017), “A Micro-Level Study of the Relationship between Experienced Corruption and Subjective Wellbeing in Africa", The Journal of Development Studies, 53(1), pp. 138-155, Dor: https://doi.org/10.1080/00220388.20 16.1187721.

Transparency International (2020), https://www.transparency.org/ [fecha de consulta: 20 de abril de 2020].

Ugalde, V. (2018), "La línea 12 en la mira de la sociología de las élites delincuentes", Gestión y Política Pública, XXVII(3), pp. 281-306, DoI: https://doi.org/10.29265/gypp. v27i3.461.

Uzodike, U. y B. Maiangwa (2012), "Boko Haram Terrorism in Nigeria: Causal Factors and Central Problematic", African Renaissance, 9(1), pp. 91-118. 
Vian, T. (2008), "Corruption and the Consequences for Public Health2, en H.K. Heggenhougen (ed.), International Encyclopedia of Public Health, Cambridge, Academic Press, pp. 26-33, Dor: https://doi.org/https://doi.org/10.1016/B978-012373960-5.00507-4. Vian, T. (2020), "Anti-corruption, Transparency and Accountability in Health: Concepts, Frameworks, and Approaches", Global Health Action, 3, Dor: https://doi.org/10.1080 /16549716.2019.1694744.

Werner, S.B. (1983), "New Directions in the Study of Administrative Corruption", Public Administration Review, 43(2), pp. 146-154, DoI: https://doi.org/10.2307/975428.

Williams, C. (2014), "Uncoupling Enterprise Culture from Capitalism”, Journal of Enterprising Communities: People and Places in the Global Economy, 8(2), pp. 111-125, DOI: https://doi.org/10.1108/JEC-08-2012-0043.

Williams, C., S. Nadin, S. Newton, P. Rodgers y J. Windebank (2013), "Explaining offthe-books Entrepreneurship: A Critical Evaluation of Competing Perspectives", International Entrepreneurship and Management Journal, 9(3), pp. 447-463, DoI: https://doi. org/10.1007/s11365-011-0185-0.

Solon Bevilacqua es graduado en Administración por la Universidade Federal do Rio Grande do Sul-Ufrgs (1999), tiene maestría en Administración por la Universidade Federal de Uberlândia-ufu (2007) y doctorado en Psicología por la Pontifícia Universidade Católica-puc (2014). Actualmente es profesor adjunto de la Faculdade de Ciências e Tecnologia de la Universidade Federal de Goiás. Profesor y coordinador de la maestría profesional en Administración Pública de la UFG. Tiene experiencia en análisis de comportamiento del consumidor y desarrollo de productos.

John Edward Neira Villena es graduado en Ingeniería Mecánica por la Universidad Católica de Santa María (1999), tiene maestría en Ingeniería Mecánica por la Universidade Federal do Rio Grande do Norte (2004) y doctorado en Ciencias e Ingeniería de Materiales por la Universidade Federal do Rio Grande do Norte (2008). Actualmente es profesor adjunto de la Faculdade de Ciências e Tecnologia de la Universidade Federal de Goiás. Tiene experiencia en el área de ingeniería de materiales, con énfasis en materiales compuestos, métodos numéricos y energías renovables y eficiencia energética, principalmente en los temas de energía eólica y solar. 